\title{
Expression of aquaporin-4 water channels in the digestive tract of the guinea pig
}

Ling Jiang ${ }^{1}, \quad$ Jian $\mathrm{Li}^{3}$, Xiaofeng Liu ${ }^{2}$, Geoffrey Burnstock ${ }^{4,5}$, Zhenghua Xiang ${ }^{2} *$

${ }^{1}$ School of optical Electrical and Computer Engineering, University of Shanghai for Science and Technology, Shanghai 200093, PR China

${ }^{2}$ Department of Neurobiology, Key Laboratory of Molecular Neurobiology, Ministry of Education, Neuroscience Research Centre of Changzheng Hospital, Second Military Medical University, Shanghai 200433, PR China

${ }^{3}$ Department of Anesthesiology, Neuroscience Research Centre, Changzheng Hospital, Second Military Medical University, Shanghai 200003, China.

${ }^{4}$ Autonomic Neuroscience Centre, University College Medical School, Rowland Hill Street, London NW3 2PF

${ }^{5}$ Department of Pharmacology, The University of Melbourne, Victoria, Australia

L Jiang, Jian Li and X Liu contributed equally to this work.

*Corresponding author. Fax: +86 21 65492132. E-mail address: zhxiang@hotmail.com (Z. Xiang). 
Abstract: Expression of the aquaporin-4 water channel (AQP4) was systematically studied in the digestive tract of the guinea pig using Western blot and immunofluorescence techniques. The results showed that AQP4 was expressed widely in different segments of the guinea pig digestive tract. AQP4-immunoreactivity (ir) was confined to parietal cells in the stomach, and absorptive and glandular epithelial cells of small and large intestine. AQP4 protein was also expressed by enteric glial cells of submucosal and myenteric ganglia and primary nerve trunks. AQP4 was expressed by both type I and type II enteric gliocytes, but not by type III or type IV enteric gliocytes, indicating that enteric gliocytes have a heterogeneous distribution in the gut wall. In addition, different patterns of AQP4 expression in the enteric nervous system of human, guinea pig, rat and mouse colon mucosa were identified: in rat and mouse AQP4 was localised to a small subpopulation of neurons; in the guinea pig AQP4 was localised to enteric glial cells; and in the human colon mucosa, AQP4 was also detected mainly in the glial cells. It has been speculated that AQP4 may be involved in water transport in the gastrointestinal tract. Its role in enteric neurons and glia is unknown, but, by analogy with the brain, AQP4 may be involved in the formation and resolution of edema.

Key Words: Aquaporin-4; enteric glia; digestive tract; guinea pig, mouse, rat, human 


\section{Introduction}

Aquaporins (AQP) consists of a family of 13 integral membrane channels (AQP0-12) that are permeable to water or to water and other small solutes bi-directionally in response to an osmotic gradient. They are distributed differently among visceral organs and have various structural and physiological characteristics (King et al. 2004; Gomes et al. 2009; Isokpehi et al. 2009; Verkman 2009). The digestive system is heavily involved in fluid transport, quantitatively second only to that of the kidney. Water transportation through the digestive system is physiologically crucial for maintaining body water homeostasis and digestive and absorptive functions. Previous studies have shown that the different types of AQP in the digestive tract have distinctive distribution patterns (Koyama et al. 1999; Laforenza 2012). Several AQP isoforms are found in gastrointestinal epithelia, with AQP1, 3, 7, 10 and 11 being the most abundantly expressed in the whole gut. On the other hand, AQP4 and 8 are located selectively in the stomach and colon, respectively (Koyama et al. 1999; Laforenza 2012).

AQP4 is a water channel predominantly found in astrocytes in the central nervous system (CNS) and is believed to play a critical role in the formation and maintenance of the blood-brain barrier and in water secretion from the brain (Manley et al., 2000, Papadopoulos et al., 2002]. Enteric glial cells have some resemblance to astrocytes, both biochemically (Jessen and Mirsky, 1983; Ruhl, 2005) and morphologically (Hanani and Reichenbach, 1994). But there are contradictory reports about AQP4 expression in the enteric nervous system. AQP4 was detected in ganglionic neurons of myenteric and submucosal plexuses, but not in enteric glial 
cells in rat and mouse digestive tracts (Thi et al., 2008). In another report, AQP4 was not detected in ganglionic neuronal bodies of chicken digestive tract, but rather in the network of fibers; this will need studies using specific markers (Yoshimura et al., 2011). Using immunofluorescence staining, we found that AQP4 was expressed in high levels in enteric glial cells in the submucosal and myenteric plexuses of guinea pig digestive tract as well as in parietal cells, absorptive and glandular epithelial cells of small and large intestine.

\section{Materials and Methods}

All experimental procedures were approved by the Institutional Animal Care and Use Committee at Second Military Medical University. Six guinea pigs (250-350g), three SD rats (200-250g) and three Kuming mice (25-30g) were used. The animals were anesthetized with sodium pentobarbitone and perfused through the aorta with $0.9 \% \mathrm{NaCl}$ solution and $4 \%$ paraformaldehyde in $0.1 \mathrm{~mol} / 1$ phosphate buffer $\mathrm{pH}$ 7.4. The digestive tracts were removed and washed with phosphate buffered saline (PBS). One end of the segment was knotted with a silk thread and fixative was injected into the lumen to fill it and the open end was also knotted with a silk thread. The fixative-filled segment was then immersed in $4 \%$ paraformaldehyde in $0.1 \mathrm{~mol} / \mathrm{l}$ phosphate buffer, $\mathrm{pH} 7.4$, for 4-6 hrs. The mucosa was discarded and the submucosal and myenteric plexuses of the gastric fundus, jejunum, ileum, and proximal and distal colon ofguinea pings, mice and rats were used as whole-mount preparations.

After perfusion fixation, some parts of guinea pig digestive tracts were immersed 
in $4 \%$ paraformaldehyde in $0.1 \mathrm{~mol} / \mathrm{l}$ phosphate buffer, $\mathrm{pH} 7.4$, for $4-6 \mathrm{hrs}$ and then transferred to $25 \%$ sucrose in PBS and kept in the solution until they sank to the bottom. Three blocks of colon mucosa from humans obtained from the Department of Pathology, Changhai Hospital of Second Military Medical University were immersed in a fixative of $4 \%$ paraformaldehyde in $0.1 \mathrm{~mol} / \mathrm{L}$ phosphate buffer, $\mathrm{pH} 7.4$ for 24 hours and then transferred to $25 \%$ sucrose in PBS and kept in the solution until they sank to the bottom. Thereafter, the tissue blocks were rapidly frozen and coronal sections $(10 \mu \mathrm{m}$ in thickness) were cut with a Leica cryostat and thaw on the slides coated with gelatin.

\section{Immunohistochemistry}

The following protocol was used for double immunostaining of AQP4 with S100ß, NF200, PGP9.5 (neuronal markers) and NeuN (neuronal nuclei marker). The preparations were washed $3 \times 5 \mathrm{~min}$ in $\mathrm{PBS}$, and then preincubated in antiserum solution 1 (10\% normal bovine serum, $0.2 \%$ Triton-X-100, $0.4 \%$ sodium azide in 0.01 mol/L PBS $\mathrm{pH} 7.2$ ) for $30 \mathrm{~min}$, followed by incubation with different combinations of AQP4 antibody (rabbit anti-human, Santa Cruz, catalog number sc-20812) diluted 1:200, S100 $\beta$ (mouse anti-rat; Chemicon) diluted 1:200, PGP9.5 and NeuN antibody (mouse anti-rat; Chemicon) diluted 1:500 in antiserum solution 2 ( $1 \%$ normal bovine serum, $0.2 \%$ Triton-X-100, $0.4 \%$ sodium azide in $0.01 \mathrm{~mol} / \mathrm{L}$ PBS $\mathrm{pH} 7.2$ ) at room temperature. Subsequently, the preparations were incubated with Cy3-conjugated donkey anti-rabbit IgG diluted 1:400 for AQP4 antibody and FITC conjugated donkey anti-mouse IgG diluted 1:200 for S100ß, NF200, NeuN antibodies 
in antiserum solution 2 for $2 \mathrm{~h}$ at room temperature. Both Cy3-conjugated donkey anti-rabbit IgG and FITC conjugated donkey anti-mouse $\operatorname{IgG}$ are specialized for multiple immunostaining from Jackson Immunoresearch Lab. All the incubations and reactions were separated by $3 \times 10$ min washes in PBS.

A negative control of omitting AQP4 primary antibody was carried out. No staining was observed in those preparations.

\section{Western blot analysis}

In order to further confirm the specificity of the AQP4 antibody and to analyse the expression levels of AQP4 in the different segments of guinea pig digestive tract, Western blot analysis was carried out. Guinea pigs were deeply anesthetized by sodium pentobarbital $(60 \mathrm{mg} / \mathrm{kg})$ and killed by decapitation. The gastric fundus, jejunum, ileum and distal colon were rapidly removed, washed with ice-cold PBS and lysed with $20 \mathrm{mM}$ Tris- $\mathrm{HCl}$ buffer, $\mathrm{pH} 8.0$, containing $1 \% \mathrm{NP}-40,150 \mathrm{mM} \mathrm{NaCl}, 1$ mM EDTA, $10 \%$ glycerol, $0.1 \%$-mercaptoethanol, $0.5 \mathrm{mM}$ dithiothreitol, and a mixture of proteinase and phosphatase inhibitors (Sigma). Protein concentration was determined by the BCA protein assay method using bovine serum albumin as standard (BCA protein assay kit from Beyotime). Protein samples $(100 \mu \mathrm{g})$ were loaded per lane, separated by SDS-PAGE (10\% polyacrylamide gels) and then were electrotransferred onto nitrocellulose membranes. The membranes were blocked with $10 \%$ nonfat dry milk in Tris-buffered saline for $1 \mathrm{~h}$ and incubated overnight at $4{ }^{\circ} \mathrm{C}$ with AQP4 antibody (rabbit anti-human, Santa Cruz) diluted 1:500 in 2\% BSA in PBS. The membranes were then incubated with alkaline phosphatase-conjugated goat 
anti-rabbit IgG (Sigma) diluted 1:1000 in 2\% BSA in PBS for $1 \mathrm{~h}$ at room temperature. The color development was performed with $400 \mu \mathrm{g} / \mathrm{ml}$ nitro-blue tetrazolium, $200 \mu \mathrm{g} / \mathrm{ml}$ 5-bromo-4-chloro-3-indolyl phosphate and $100 \mathrm{mg} / \mathrm{ml}$ levamisole in TSM2 (0.1 mol/ 1 Tris-HCl2 buffer, $\mathrm{pH} 9.5,0.1 \mathrm{~mol} / 1 \mathrm{NaCl}$ and $0.05 \mathrm{~mol} / 1 \mathrm{MgCl} 2)$ in the dark. Bands were scanned using a densitometer (GS-700; Bio-Rad Laboratories).

\section{Photomicroscopy}

Images were taken with the Nikon digital camera DXM1200 (Nikon, Japan) attached to a Nikon Eclipse E600 microscope (Nikon). Images were imported into a graphics package (Adobe Photoshop).

\section{Results}

Western blotting, performed on tissue extracts derived from the guinea pig gastric fundus, jejunum, ileum and distal colon (Fig. 1), assessed the specificity of the polyclonal AQP4 antibody and expression levels of AQP4 in different segments of the guinea pig digestive tract. An immunoreactive band was detected at about $34 \mathrm{kDa}$ that corresponded to the molecular weight of AQP4 (Fig. 1 lane 1, 2, 3 and 4), no other bands were detected. The highest level of AQP4 protein was detected in the gastric body, the expression level of AQP4 protein in other segments was quite similar (Fig. 1 lane 2, 3 and 4). Omitting the AQP4 primary antibody resulted in the absence of the band (Fig. 1 lane 5, protein specimen from guinea pig gastric fundus), indicating that the antibodies detected the appropriate antigen sequence.

The expression pattern of AQP4 protein detected with immunofluorescence was similar with that detected with Western blotting technique. AQP4-immunoreactivity 
(-ir) was demonstrated in all the segments of guinea pig digestive tract. Strong AQP4-ir was found in the gastric body and weak to moderate immunostaining was also detected in other segments (Figure 2).

In the gastric fundus sections, very strong AQP4 immunostaining was detected in the bottom of gastric glands, and weak to moderate AQP4-ir in the neck. These AQP4-ir cells were oval in shape with morphological characteristics of parietal cells. In the bottom of the gastric gland, AQP4-ir was concentrated in the basolateral membrane of parietal cells. In the neck of the gastric gland, AQP4-ir was localized in the whole cytoplasm (Figure 2B1, B2). In the small intestine sections, weak to moderate AQP4-ir was detected in epithelial cells of villi and intestinal glands. AQP4 immunostaining was denser in the striated border and basal membrane of epithelial cells (Figure 2B, C, E). In the distal colon sections, strong AQP4-ir was detected in the basal and bi-lateral membranes of the colon epithelium and intestinal gland epithelium (Figure 2F). Strong AQP4-ir was also localized in the submucosal and myenteric plexuses in the gastric fundus, jejunum, ileum and distal colon (Figure 2A, $\mathrm{C}$ and F, Figure 3). In the sections of the stomach and intestines, strong AQP4-ir was detected in the submucosal and myenteric plexuses. AQP4-ir was mainly localised to the ganglia of submucosal and myenteric plexuses, being almost undetectable in the intramuscular neve trunks and under the mucosal epithelium (Figure 3).

In order to confirm the cell types with AQP4-ir in the submucosal and myenteric plexuses, double labeling immunofluorescence and whole-mount preparations of submucosal and myenteric plexuses of stomach, jejunum, ileum and 
distal colon were examined. The results showed that AQP4-ir coexisted with S100ß, but did not coexist with NeuN or NF200.

In whole-mount preparations of guinea pig stomach, small AQP4-ir cells with thin and short processes were detected in the ganglia of stomach myenteric plexus. S100ß-ir was mainly localized in cell bodies and nuclei in the stomach myenteric plexus. The merged image showed that all AQP4-ir cells were also immunoreactive for S100 $\beta$, but only a small subset of S100 $\beta$-ir cells (about 30\%) were immunoreactive for AQP4. No AQP4-ir cells were detected in the nerve trunks that connect ganglia and intramuscular nerve trunks (Figure 4). In whole-mount preparations of guinea pig jejunum and ileum, the distribution pattern of AQP4-ir cells and processes was quite similar. AQP4-ir cells and processes were detected in the ganglia and primary nerve trunks of ganglia, but not in the secondary nerve trunk network. In ganglia, AQP4-ir cells and processes comprised a fine network around the ganglionic neuronal bodies where no AQP4-ir was detected (Figure 4A, B, C). The distribution pattern of S100 $\beta$-ir cells and processes in the ganglia was almost the same as that of AQP4-ir. The images of AQP4-ir cells and processes were almost merged with that of S100ß-ir. In the primary nerve trunks of ganglia, only some S100 $\beta$-ir cells and processes were also immunoreactive for AQP4 (Figure 4D, E, F). In whole-mount preparations of guinea pig proximal and distal colon, the distribution pattern of AQP4-ir cells and processes was also similar to that of jejunum and ileum (Figure 5).

In whole-mount preparations of submucosal plexus of jejunum, ileum and colon, AQP4-ir cells and processes also comprise a fine network around the submucosal 
ganglionic neuronal bodies. AQP4-ir was also mainly detected in the ganglia and primary nerve trunks, but not in the secondary nerve trunks (Figure 6). S100ß-ir was detected in enteric glial bodies and processes. The distribution pattern of S100ß-ir was almost the same as that of AQP4-ir. All AQP4-ir cells and processes were also immunoreactive for $\mathrm{S} 100 \beta$, but $\mathrm{S} 100 \beta$-ir cells and processes in the secondary nerve trunks of the submucosal plexus were not immunoreactive for AQP4 (Figure 6). In order to obtain the precise localization of AQP4-ir, some whole-mount specimens of myenteric and submucosal plexuses were scanned using a laser confocal microscope (Leica SP2). In these specimens, AQP4-ir was precisely localized in the enteric glia using S100ß-ir, but not in the ganglionic neurons (Figure 5 and Figure 6 D, E, F). Some images of guinea pig myenteric and submucosal plexuses immunostained with both AQP4 and S100ß and low magnification images are shown in supplementary Figures 1 and 2.

Double labeling immunofluorescence showed that AQP4-ir did not coexist with the neuronal markers NeuN or NF200 in whole-mount preparations of different segments of guinea pig digestive tract, as exemplified in the myenteric plexus of the ileum (Figure7A, B, C) and distal colon (Figure 7D, E, F).

Previous data has shown that AQP4 is exclusively expressed by a small subpopulation of neurons in the mouse and rat enteric nervous system (Thi et al., 2008), which is different from the results of this study. In order to clarify this issue, whole-mount preparations of myenteric and submucosal plexus from mouse and rat were also studied with AQP4 antibody. The present results are similar to those of the 
previous report (Thi et al., 2008): AQP4 was exclusively expressed in a small subpopulation of neurons in the myenteric and submucosal plexus of mouse and rat (supplementary Figure 3).

We also studied the localization of AQP4-ir in the human colon mucosa and found that AQP4 was mainly expressed in astrocyte-like cells. These AQP4-ir cells were also immunoreactive for S100ß. These AQP4-ir cells and their processes were numerous around the colon glands (Figure 8).

\section{Discussion}

Using Western blot and single and double labeling immunofluorescence techniques, expression of AQP4 protein was systematically studied in the digestive tract of the guinea pig. The results showed that AQP4 was expressed widely in absorptive and glandular epithelial cells and enteric glial cells of different segments of guinea pig digestive tract. To our knowledge, this is the first time that enteric glial cells have been shown to express an aquaporin. In addition, different patterns of AQP4 expression in the enteric nervous system in guinea pig, rat, and mouse were found in this study.

Thirteen AQPs have been divided into three groups of orthodox AQPs, aquaglyceroporins and unorthodox aquaporins according to their functional characteristics (Agre et al., 2002; Ishibashi, 2006; Rojek et al., 2008). AQP4 belongs to the orthodox group of AQPs. In this study, AQP4-ir was detected in parietal cells, absorptive epithelia and glandular epithelia. These results further extend the previous data (Misaka et al., 1996; Koyama et al., 1999; Fujita et al., 1999; Huang et al., 2003; 
Ma and Verkman 1999; Laforenza 2012). AQP4 was also identified in the chief cells of human stomach (Misaka et al., 1996), but was not identified in guinea pig stomach in this study. AQP4 was found to be concentrated in the basolateral membranes of parietal cells, colon epithelia and glandular cells, and basal and striated border membranes of small intestinal epithelia. This pattern of AQP4 expression is different from other AQPs that were found mainly in the superficial portion of rat colon mucosa (Friger et al., 1995 and Laforenza et al., 2005). The intestinal epithelia have two pathways for water transport: the paracellular and the transcellular routes, the former is through the spaces between cell junctions and the latter is through apical and basolateral cell membranes (Field, 2003; Barrett, 2008; Rosenthal et al., 2010; Fischbarg, 2010). The results from this study imply that AQP4 may be involved in water transport through the transcellular route in the guinea pig digestive tract.

Aquaporins have also been detected in the central and peripheral nervous systems. These water channel proteins are expressed at high levels in astrocytes of the CNS (Nielsen et al., 1997; Amiry-Moghaddam and Ottersen, 2003). AQP4 is localized in astrocytic end-feet membranes that contact capillaries and pia where the exchange of water between the brain and extracerebral liquids takes place. It was therefore suggested that AQP4 may be involved in the regulation of water homeostasis in the central nervous system (Amiry-Moghaddam and Ottersen, 2003; Nicchia et al., 2004). An AQP4 knock-out mouse study indicated that AQP4 may be involved in the formation and resolution of brain and spinal cord edema (Manley et al., 2000; Papadopoulos et al., 2004). Compared to normal mice, AQP4 knock-out mice exhibit 
reduced brain edema and neurologic improvement following ischemic brain injury (Manley et al., 2004; Verkman 2006).

Compared to the studies of AQP4 in the central nervous system, expression and function of AQP4 in the enteric nervous system have not been fully investigated. In this study, we showed that AQP4 was exclusively expressed in enteric glial cells of intraganglia and primary fiber tracts in submucosal and myenteric plexus of guinea pig, but did not coexist with either NeuN or NF200, which implies that AQP4 may play a physiological role in enteric glia of guinea pig.

Enteric glial cells are a unique class of peripheral glial cells within the gastrointestinal tract. The majority of enteric glial cells are detected in enteric ganglia in the myenteric and submucosal plexus of the enteric nervous system. Enteric glial cells are also detected outside the enteric nervous system, within muscles and in the lamina propria of the mucosa. Enteric glial cells are divided into four types based on morphological differences: type I or "protoplasmic" enteric gliocytes within enteric ganglia, resembling protoplasmic astrocytes of the CNS; type II or "fibrous" enteric gliocytes within nerve trunks that connect ganglia, resembling fibrous astrocytes of white matter in the CNS; type III or "mucosal" enteric gliocytes, having several long-branched processes and located under enteric epithelia; type IV or “intramuscular" enteric gliocytes, elongated glial cells running with nerve fibers in muscles (Hanani, and Reichenbach 1994; Gulbransen and Sharkey 2012). According to this classification of enteric gliocytes, AQP4 was found to be expressed by type I and type II enteric gliocytes, but not by type III or type IV enteric gliocytes, because 
in this study AQP4-ir was detected in ganglia and nerve trunk, and not within muscles or lamina propria of the mucosa. The fact that AQP4 was only expressed by type I and II gliocytes further illustrates the heterogeneous distribution of enteric gliocytes within the gut wall.

Previous data showed that AQP4 is exclusively expressed in a small subpopulation of neurons in the mouse and rat enteric nervous system (Thi et al., 2008), the results of which were confirmed in the present study, in contrast to the guinea pig where AQP4 was exclusively expressed in glial cells. In the human colon mucosa, AQP4 was also detected mainly in glial cells. These results showed that there are different patterns of AQP4 expression in the enteric nervous system of mice, rats, guinea pigs and humans.

Previous data has shown that AQP1, but not AQP4, was identified in glial cells of human pancreatic nerve plexuses and in the submucosal and myenteric nerve plexuses of human esophagus (Gao et al., 2006). This previous data differs from this report, where we showed that AQP4 was expressed in glial cells of the guinea pig nervous system. Both AQP1 and AQP4 belong to the orthodox group of AQPs that have similar functional characteristics. Thus, it is possible that different aquaporins may play similar roles in different species.

In summary, the expression of AQP4 was systematically studied in the digestive tract of the guinea pig. The results showed that AQP4 was expressed widely in different segments of the guinea pig digestive tract. AQP4-ir was confined to parietal cells in the stomach, and absorptive and glandular epithelial cells of the small and 
large intestine. AQP4 was also expressed in enteric glial cells of submucosal and myenteric ganglia and primary nerve trunks. AQP4 was expressed by type I and type II enteric gliocytes, but not by type III or type IV enteric gliocytes. The fact that AQP4 was only expressed by type I and II gliocytes further illustrates the heterogeneous distribution of enteric gliocytes along the gut wall. In addition, this study showed that there were different patterns of AQP4 expression in the enteric nervous system of mice, rats, guinea pigs and humans. 


\section{Acknowledges}

The authors thank Dr. Gillian E. Knight for her excellent editorial assistance.

This work was supported by 973 Program (2011CB504401 to Z. Xiang) and the National Natural Science Foundation of PR China (30970918 to Z. Xiang). 


\section{References}

Agre P, King LS, Yasui M, Guggino WB, Ottersen OP, Fujiyoshi Y, Engel A and Nielsen S (2002) Aquaporin water channels--from atomic structure to clinical medicine. J Physiol 542: 3-16

Amiry-Moghaddam M and Ottersen OP (2003) The molecular basis of water transport in the brain. Nat Rev Neurosci 4: 991-1001

Barrett KE (2008) New ways of thinking about (and teaching about) intestinal epithelial function. Adv Physiol Educ 32: 25-34

Field M (2003) Intestinal ion transport and the pathophysiology of diarrhea. J Clin Invest 111: 931-43

Fischbarg J (2010) Fluid transport across leaky epithelia: central role of the tight junction and supporting role of aquaporins. Physiol Rev 90:1271-90

Frigeri A, Gropper MA, Turck CW, Verkman AS (1995) Immunolocalization of the mercurial-insensitive water channel and glycerol intrinsic protein in epithelial cell plasma membranes. Proc Natl Acad Sci U S A 92(10):4328-31.

Fujita A, Horio Y, Nielsen S, Nagelhus EA, Hata F, Ottersen OP and Kurachi Y (1999) High-resolution immunogold cytochemistry indicates that AQP4 is concentrated along the basal membrane of parietal cell in rat stomach. FEBS Lett 459: 305-9

Gao H, He C, Fang X, Hou X, Feng X, Yang H, Zhao X, Ma T (2006) Localization of aquaporin-1 water channel in glial cells of the human peripheral nervous system. Glia. 2006 53(7):783-7

Gomes D, Agasse A, Thiebaud P, Delrot S, Geros H and Chaumont F (2009) 
Aquaporins are multifunctional water and solute transporters highly divergent in living organisms. Biochim Biophys Acta 1788:1213-28

Gulbransen BD and Sharkey KA (2012) Novel functional roles for enteric glia in the gastrointestinal tract. Nat Rev Gastroenterol Hepatol 9: 625-32

Hanani M and Reichenbach A (1994) Morphology of horseradish peroxidase (HRP)-injected glial cells in the myenteric plexus of the guinea-pig. Cell Tissue Res 278:153-60

Huang Y, Tola VB, Fang P, Soybel DI and Van Hoek AN (2003) Partitioning of aquaporin-4 water channel mRNA and protein in gastric glands. Dig Dis Sci 48: 2027-36

Ishibashi K (2006) Aquaporin superfamily with unusual npa boxes: S-aquaporins (superfamily, sip-like and subcellular-aquaporins). Cell Mol Biol (Noisy-le-grand) $52: 20-7$

Isokpehi RD, Rajnarayanan RV, Jeffries CD, Oyeleye TO and Cohly HH (2009) Integrative sequence and tissue expression profiling of chicken and mammalian aquaporins. BMC Genomics 10 Suppl 2: S7

Jessen KR and Mirsky R (1983) Astrocyte-like glia in the peripheral nervous system: an immunohistochemical study of enteric glia. J Neurosci 3: 2206-18

King LS, Kozono D and Agre P (2004) From structure to disease: the evolving tale of aquaporin biology. Nat Rev Mol Cell Biol 5: 687-98

Koyama Y, Yamamoto T, Tani T, Nihei K, Kondo D, Funaki H, Yaoita E, Kawasaki K, Sato N, Hatakeyama K, and Kihara I (1999) Expression and localization of 
aquaporins in rat gastrointestinal tract. Am J Physiol 276(3 Pt 1):C621-7

Laforenza U, Cova E, Gastaldi G, Tritto S, Grazioli M, LaRusso NF, Splinter PL, D'Adamo P, Tosco M, Ventura U (2005) Aquaporin-8 is involved in water transport in isolated superficial colonocytes from rat proximal colon. J Nutr 135(10):2329-36

Laforenza U (2012) Water channel proteins in the gastrointestinal tract. In Mol Aspects Med 33(5-6):642-50

Ma T and Verkman AS (1999) Aquaporin water channels in gastrointestinal physiology. J Physiol 517 ( Pt 2): 317-26

Manley GT (2000) Aquaporin-4 deletion in mice reduces brain edema after acute water intoxication and ischemic stroke. Nature Med 6:159-163

Manley GT, Binder DK, Papadopoulos MC and Verkman AS (2004) New insights into water transport and edema in the central nervous system from phenotype analysis of aquaporin-4 null mice. Neuroscience 129:983-91

Manley GT, Fujimura M, Ma T, Noshita N, Filiz F, Bollen AW, Chan P and Verkman AS (2000) Aquaporin-4 deletion in mice reduces brain edema after acute water intoxication and ischemic stroke. Nat Med 6: 159-63

Misaka T, Abe K, Iwabuchi K, Kusakabe Y, Ichinose M, Miki K, Emori Y, Arai S (1996). A water channel closely related to rat brain aquaporin 4 is expressed in acid- and pepsinogen-secretory cells of human stomach. FEBS Lett. 381(3):208-12

Nicchia GP, Nico B, Camassa LM, Mola MG, Loh N, Dermietzel R, Spray DC, Svelto 
M and Frigeri A (2004) The role of aquaporin-4 in the blood-brain barrier development and integrity: studies in animal and cell culture models. Neuroscience 129: 935-45

Nielsen S, Nagelhus EA, Amiry-Moghaddam M, Bourque C, Agre P and Ottersen OP (1997) Specialized membrane domains for water transport in glial cells: high-resolution immunogold cytochemistry of aquaporin-4 in rat brain. $\mathrm{J}$ Neurosci 17:171-80

Papadopoulos MC, Krishna S and Verkman AS (2002) Aquaporin water channels and brain edema. Mt Sinai J Med 69: 242-8

Papadopoulos MC, Manley GT, Krishna S and Verkman AS (2004) Aquaporin-4 facilitates reabsorption of excess fluid in vasogenic brain edema. FASEB J $18: 1291-3$

Rojek A, Praetorius J, Frokiaer J, Nielsen S and Fenton RA (2008) A current view of the mammalian aquaglyceroporins. Annu Rev Physiol 70:301-27

Rosenthal R, Milatz S, Krug SM, Oelrich B, Schulzke JD, Amasheh S, Gunzel D and Fromm M (2010) Claudin-2, a component of the tight junction, forms a paracellular water channel. J Cell Sci 123:1913-21

Ruhl A (2005) Glial cells in the gut. Neurogastroenterol Motil 17: 777-90

Thi MM, Spray DC and Hanani M (2008) Aquaporin-4 water channels in enteric neurons. J Neurosci Res 86:448-56

Verkman AS (2006) Roles of aquaporins in kidney revealed by transgenic mice. Semin Nephrol 26:200-8 
Verkman AS (2009) Aquaporins: translating bench research to human disease. J Exp Biol 212:1707-15

Yoshimura K, Sugiura K, Ohmori Y, Aste N and Saito N (2011) Immunolocalization of aquaporin-4 in the brain, kidney, skeletal muscle, and gastro-intestinal tract of chicken. Cell Tissue Res 344: 51-61 


\section{Figure legends}

Figure 1. Western blot analysis of expression levels of AQP4 in the digestive tract of the guinea pig (A and B). (A) An immunoreactive band was detected at about $34 \mathrm{kDa}$. The highest level of AQP4 protein was detected in the stomach. Omitting the AQP4 primary antibody resulted in the absence of the band (lane control). (B) Ratio of AQP4 and actin immunostaining signals in different segments of the guinea pig digestive tract. These data suggest that the AQP4 antibody detected the appropriate antigen sequence.

Figure 2. Localization of AQP4-immunoreactivity (ir) in sections of different segments of the guinea pig digestive tract. (A) AQP4-ir in the gastric fundus. Strong AQP4-ir was detected at the bottom of gastric glands, and weak to moderate AQP4-ir in the neck. In the bottom part of the gastric gland, AQP4-ir was concentrated in the basolateral sides of cells (B1). In the neck part, AQP4-ir was distributed throughout the cytoplasm (B2). B1 and B2 are high magnifications of the areas indicated by + and * in A, respectively. C, D and E show AQP4-ir in the jejunum $(\mathrm{C}$ and $\mathrm{D})$ and ileum (E) sections. Weak to moderate AQP4-ir was detected in epithelial cells of villi and intestinal glands. AQP4 immunostaining was deeper in the striated border (indicated by arrows in $\mathrm{C}$ and $\mathrm{E}$ ) and basal membrane of glandular epithelial cells (indicated by an arrow in D). Note that strong AQP4-ir was detected in the submucosal plexus (SMP) as indicated by an arrow in C. F shows AQP4-ir in the distal colon section. Strong AQP4-ir was detected in the basal and bi-lateral 
membranes of the colon epithelium and gland epithelium. Scale bars $=80 \mu \mathrm{m}$ in A, $40 \mu \mathrm{m}$ in B1, B2, C and D.

Figure 3. Colocalization of AQP4-ir and S100ß-ir in the submucosal and myenteric plexuses. A, B and C show colocalization (C) of AQP4-ir (A) and S100ß-ir (B) in the ileum section. Note that AQP4-ir was mainly localised to the myenteric plexuses and was not detectable in the intramuscular nerve trunks, although S100 $\beta$-ir positive cells were clearly shown in intramuscular nerve trunks, as indicated by an arrow in B. D shows AQP4-ir in the gastric gland and myenteric plexus. E shows colocalization of AQP4-ir (red) and S100ß-ir (green) in the same area of D. Note that S100ß-positive cells were detected widely in the mucosal and muscular layers, but not in gland cells. Colocalization of AQP4-ir and S100 $\beta$-ir was only detected in the myenteric plexus. S100ß-positive cells without AQP4-ir in the mucosal (an arrow head) and myenteric (an arrow) layers were clearly detected. F shows colocalization of AQP4-ir (red) and S100ß-ir (green) in the submucosal layer of jejunum. Note that colocalization of AQP4-ir and S100ß-ir was only detected in the submucosal plexus, not in the other areas. All scale bars $=40 \mu \mathrm{m}$.

Figure 4. Colocalization of AQP4-ir and S100ß-ir in a whole-mount preparation of stomach and small intestine myenteric plexus. A, B and C show colocalization (C) of AQP4-ir (A) and S100ß-ir (B) in the stomach myenteric plexus. Note that only a few S100ß-ir cells also expressed AQP4-ir. An arrow indicates a typical cell showing 
colocalization of AQP4-ir and S100ß-ir in A, B and C. An arrow head indicates an S100ß-ir-positive cell without AQP4-ir in B and C. D, E and F show colocalization (F) of AQP4-ir (D) and S100ß-ir (E) in the myenteric plexus of jejunum. Note that almost all the AQP4-ir cells or processes were also immunoreactive for S100 $\beta$ in the ganglia and primary nerve trunks; S100 $\beta$-ir cells in the intramuscular nerve trunks were not found to express AQP4- ir, as indicated by an arrow in F. All scale bars $=40 \mu \mathrm{m}$ in.

Figure 5. Confocal microscopic analysis of AQP4-ir and S100ß-ir in a whole-mount preparation of myenteric plexus of the distal colon. A, B and C show colocalization (C) of AQP4-ir (A) and S100ß-ir (B) in the myenteric plexus. Note that almost all the AQP4-ir cells or processes were also immunoreactive for $\mathrm{S} 100 \beta$ in the myenteric plexus; an arrow in A, B and C indicates a typical double labelled cell with AQP4-ir and S100 $\beta$-ir. scale bars $=40 \mu \mathrm{m}$ in $\mathrm{A}, \mathrm{B}, 20 \mu \mathrm{m}$ in $\mathrm{C}$.

Figure 6. Colocalization of AQP4-ir and S100 $\beta$-ir in a whole-mount preparation of submucosal plexus of ileum and distal colon. A, B and C show colocalization (C) of AQP4-ir (A) and S100ß-ir (B) in ileum submucosal plexus. Note that almost all the AQP4-ir cells were also immunoreactive for $\mathrm{S} 100 \beta$ in the submucosal plexus, but many cells with S100ß-ir were not labeled with AQP4-ir, as indicated by an arrow in C. D, E and F show confocal microscopic analysis of colocalization (F) of AQP4-ir (D) and S100ß-ir (E) in the submucosal plexus of distal colon. Note that almost all the AQP4-ir cells were also immunoreactive for S100 $\beta$ in the submucosal plexus, but 
some cells with S100ß-ir were not labeled with AQP4-ir, as indicated by an arrow in F.

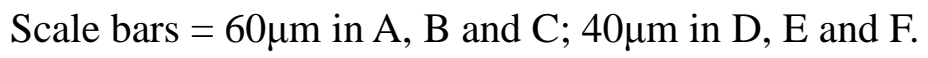

Figure 7. AQP4-ir cells were not labeled with the neuronal markers NeuN or NF200 in the myenteric plexus of the guinea pig digestive tract. A shows AQP4-ir cells and processes in the myenteric plexus of ileum, B shows NeuN-ir neurons in the same field of $\mathrm{A}$, and $\mathrm{C}$ is the merged image of $\mathrm{A}$ and $\mathrm{B}$. Note that AQP4-ir cells were not labeled with NeuN-ir. D shows AQP4-ir cells and processes in the myenteric plexus of distal colon, E shows NF200-ir neurons and processes in the same field of D, F is the merged image of D and E. Note that AQP4-ir cells were not labeled with NF200-ir. All scale bars $=40 \mu \mathrm{m}$.

Figure 8. Colocalization of AQP4-ir and S100ß-ir in a section of human colon mucosa. A shows AQP4 expression, B shows S100 $\beta$ expression in the same field of A, C shows counter-staining with DAPI, and D is the merged image from A, B and C. Note that AQP4-ir was mainly localized in cells that were also immunoreactive for S100 $\beta$; weak AQP4-ir was also detected in epithelial cells of colon glands. All scale bars = $40 \mu \mathrm{m}$. 
A

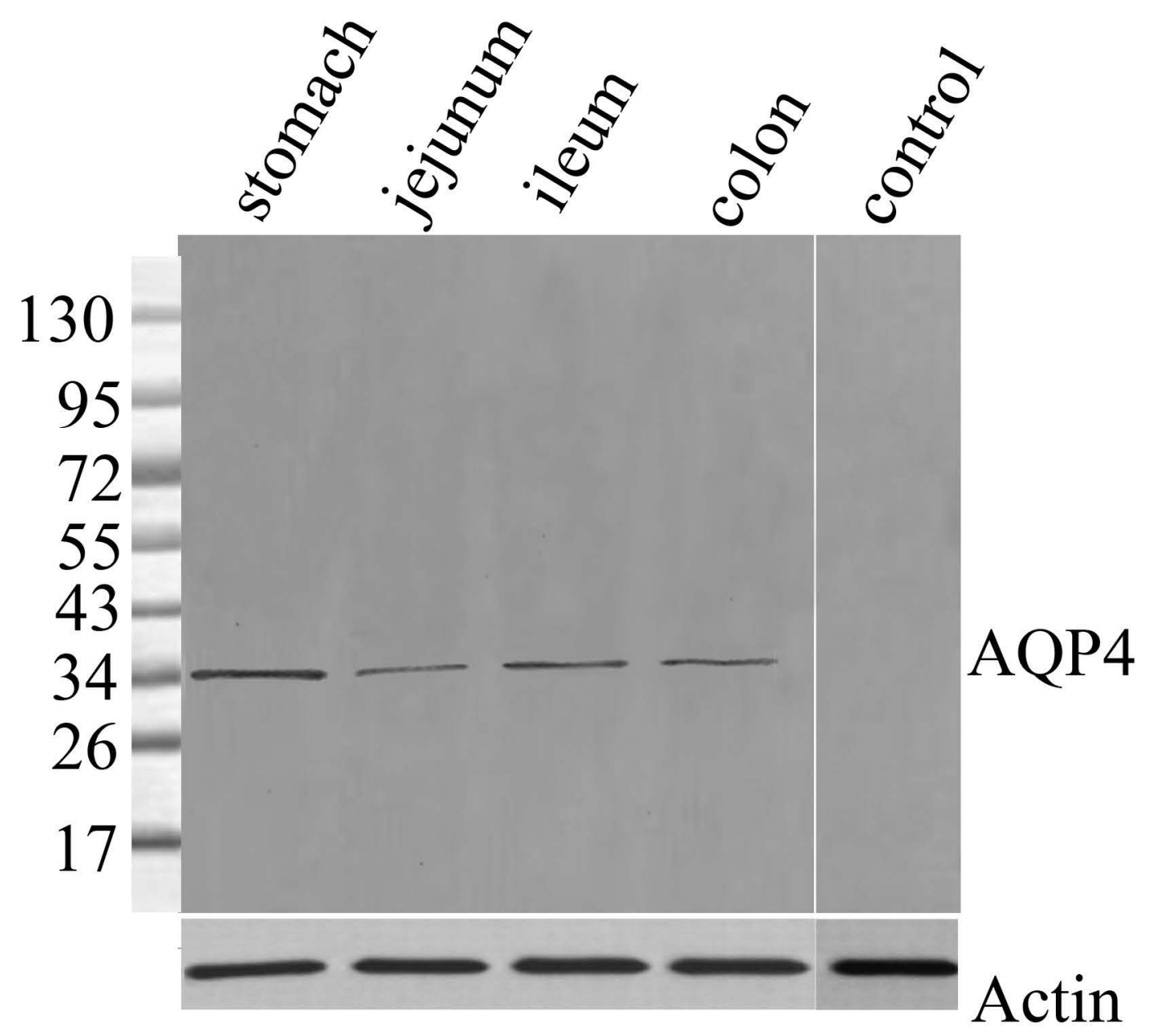

B

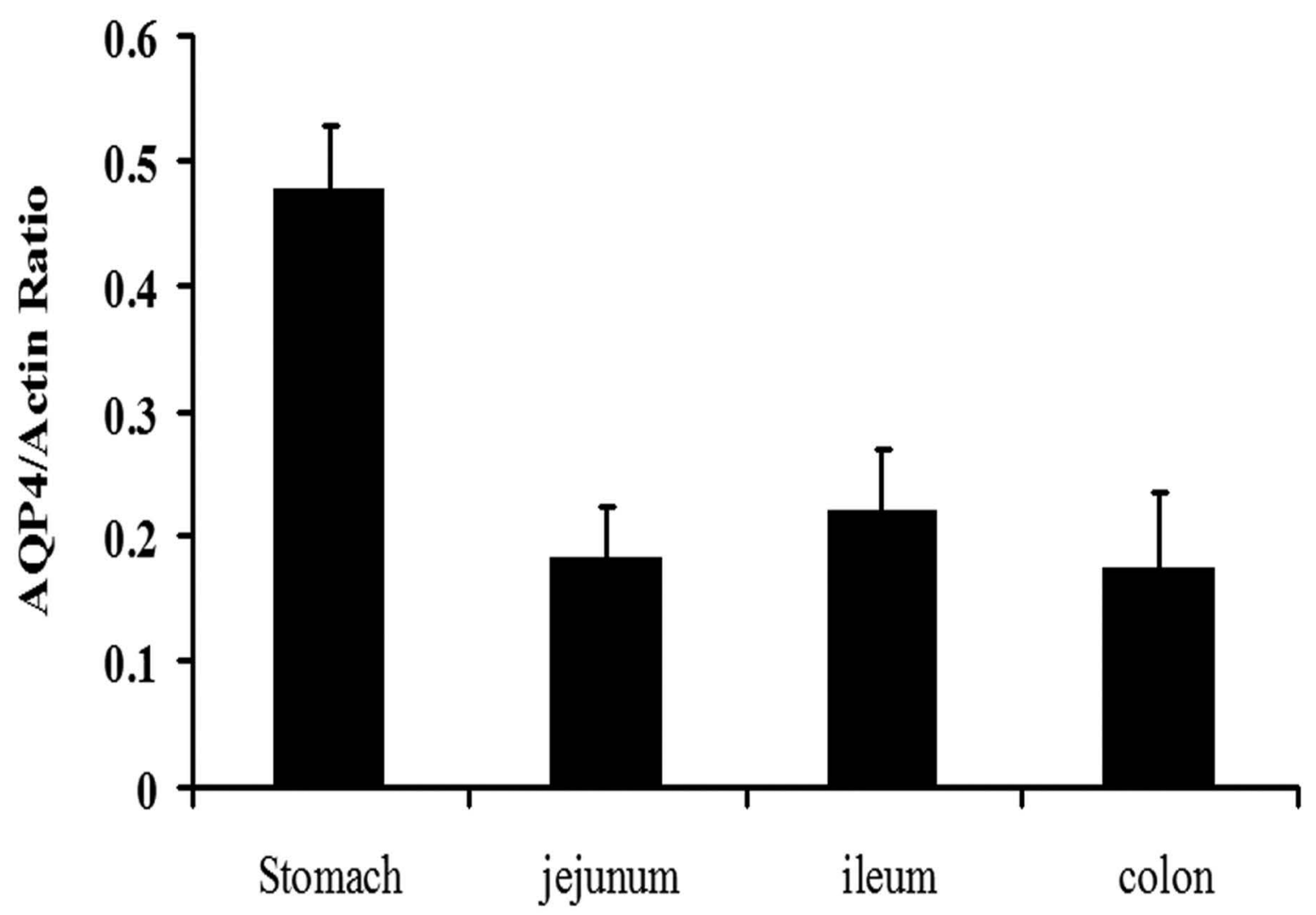


A

D

$*$,

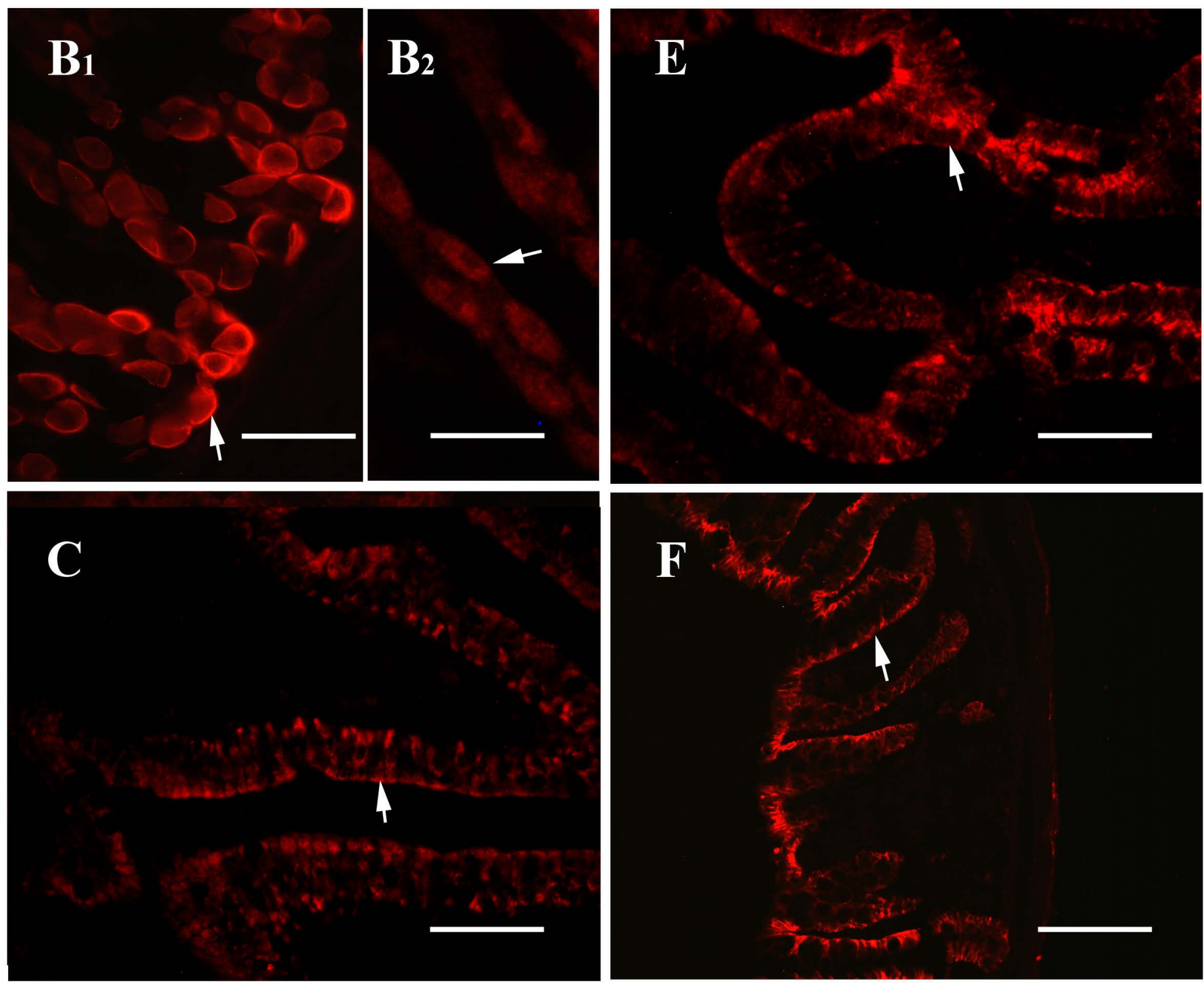




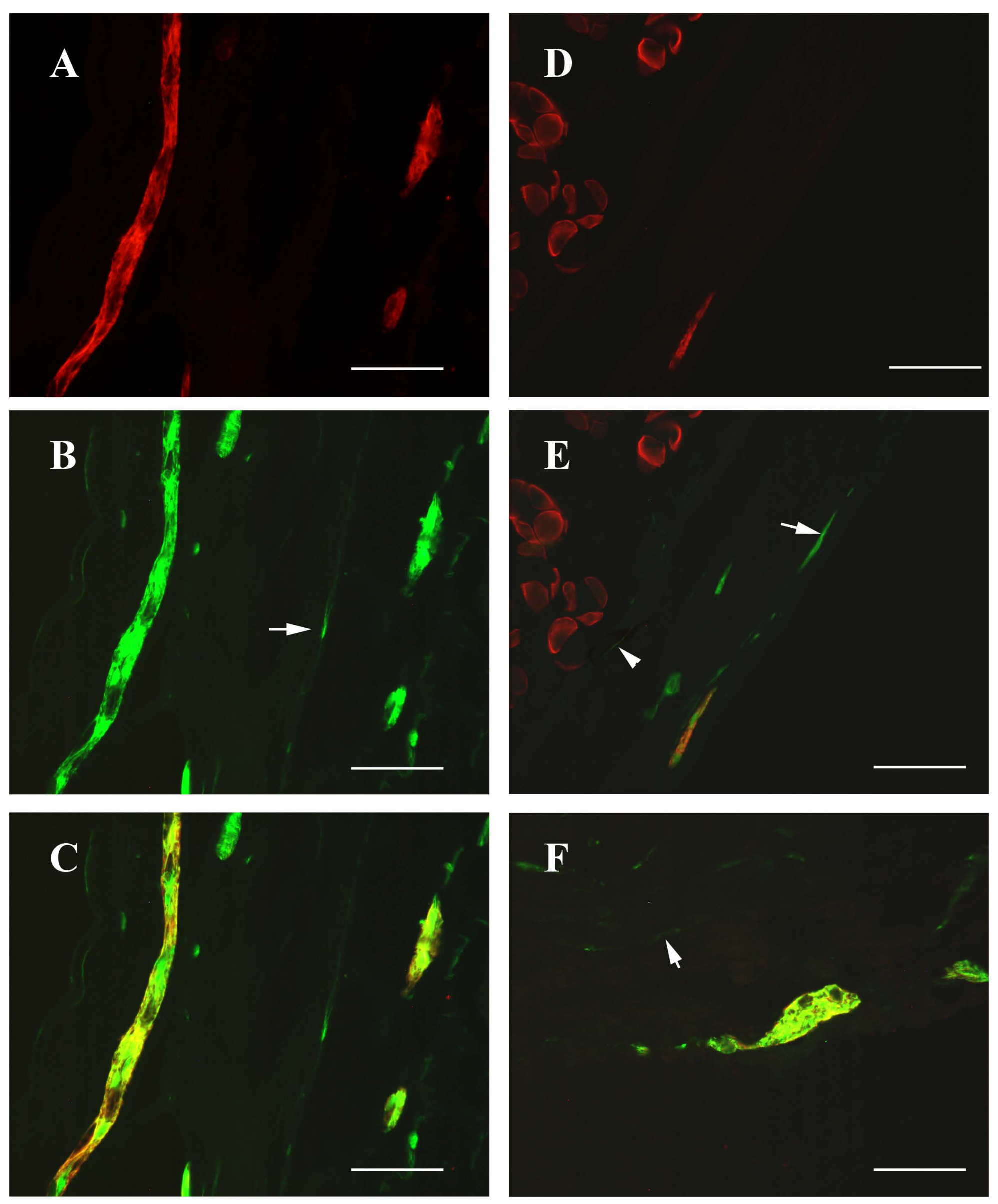


A

D

4
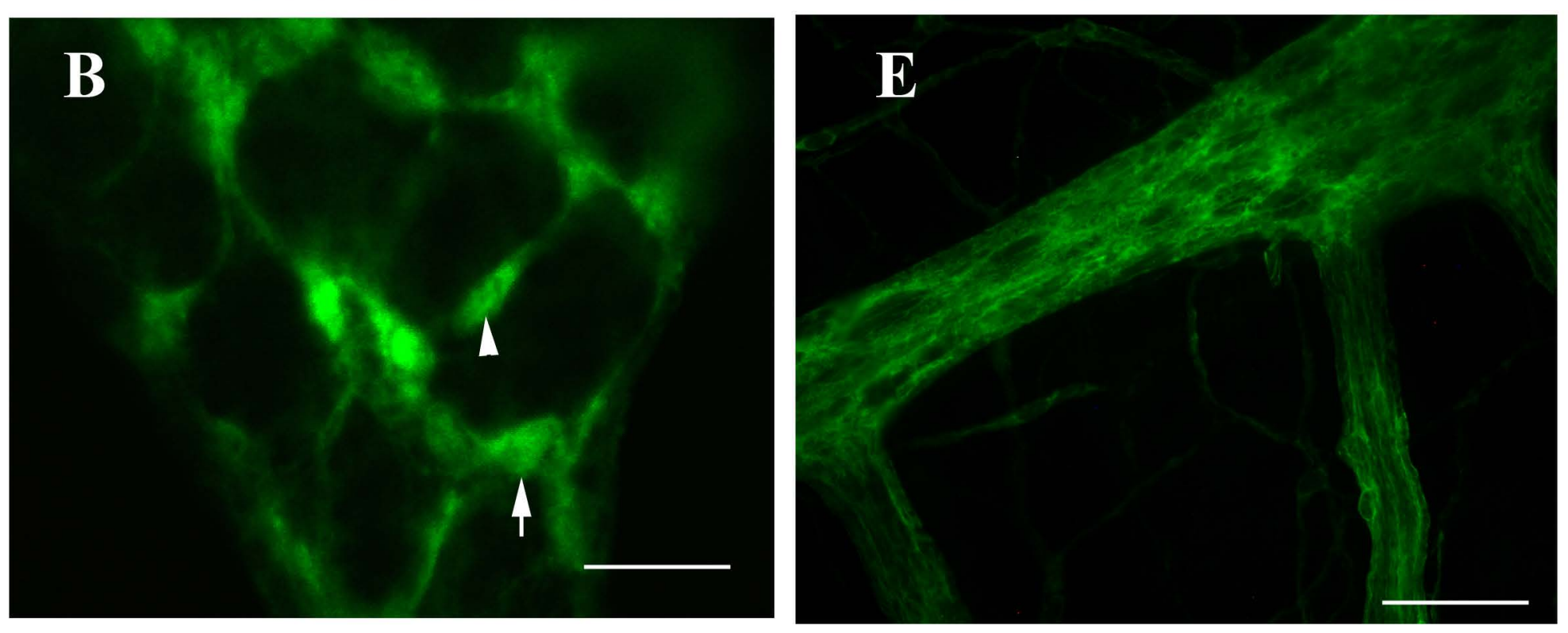

C

F

$\Lambda$

4

$\uparrow$ 


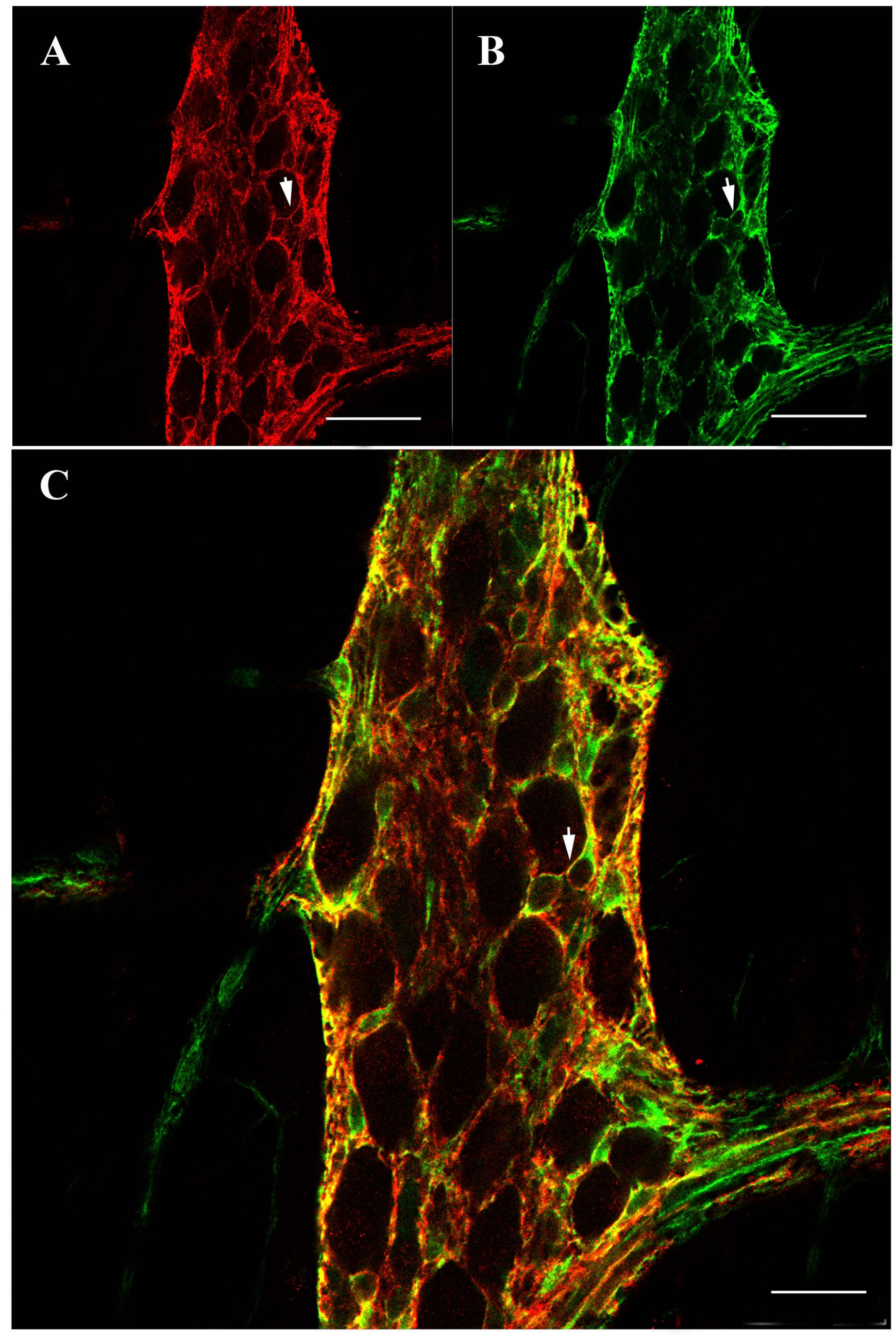



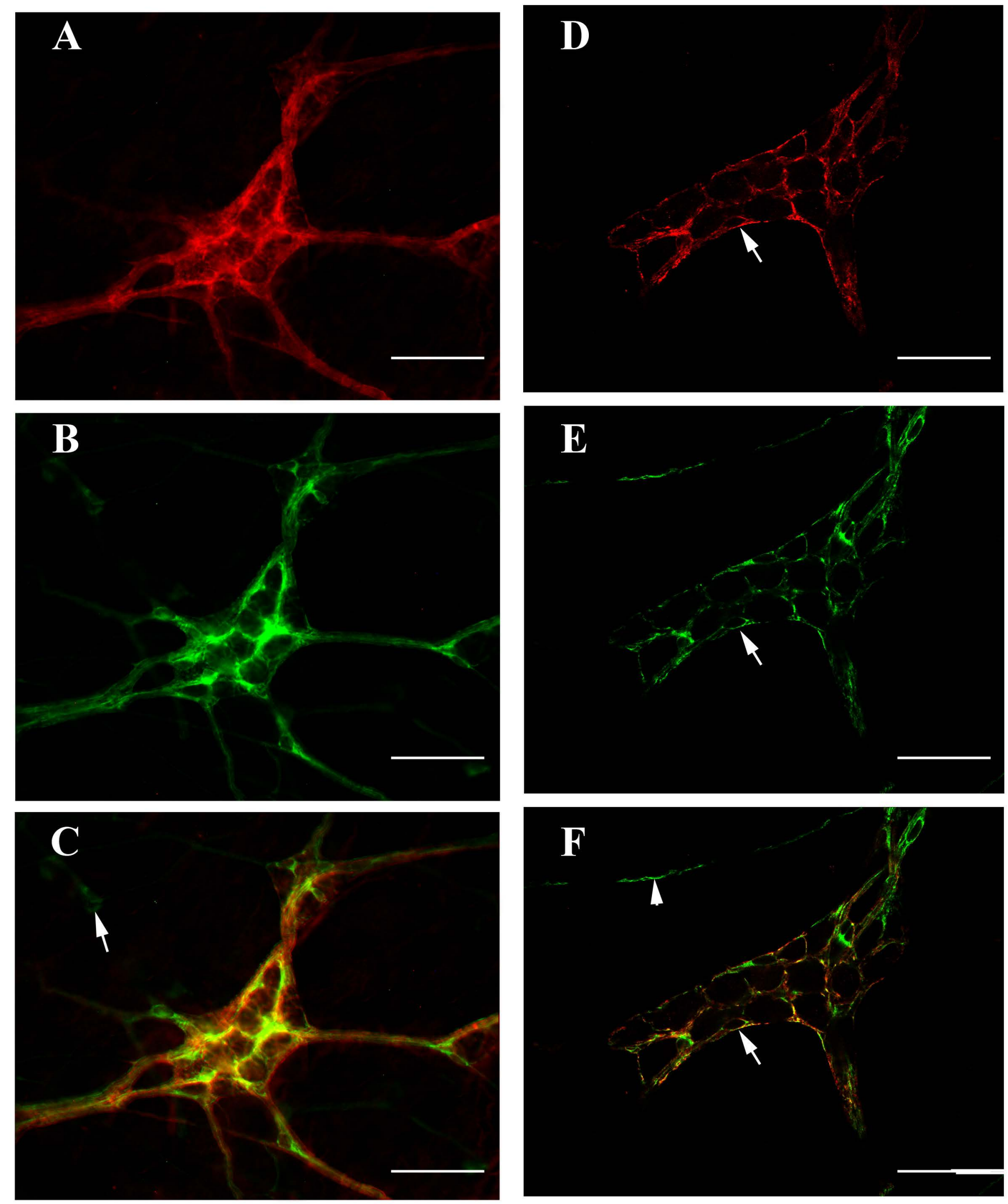
A

D

1
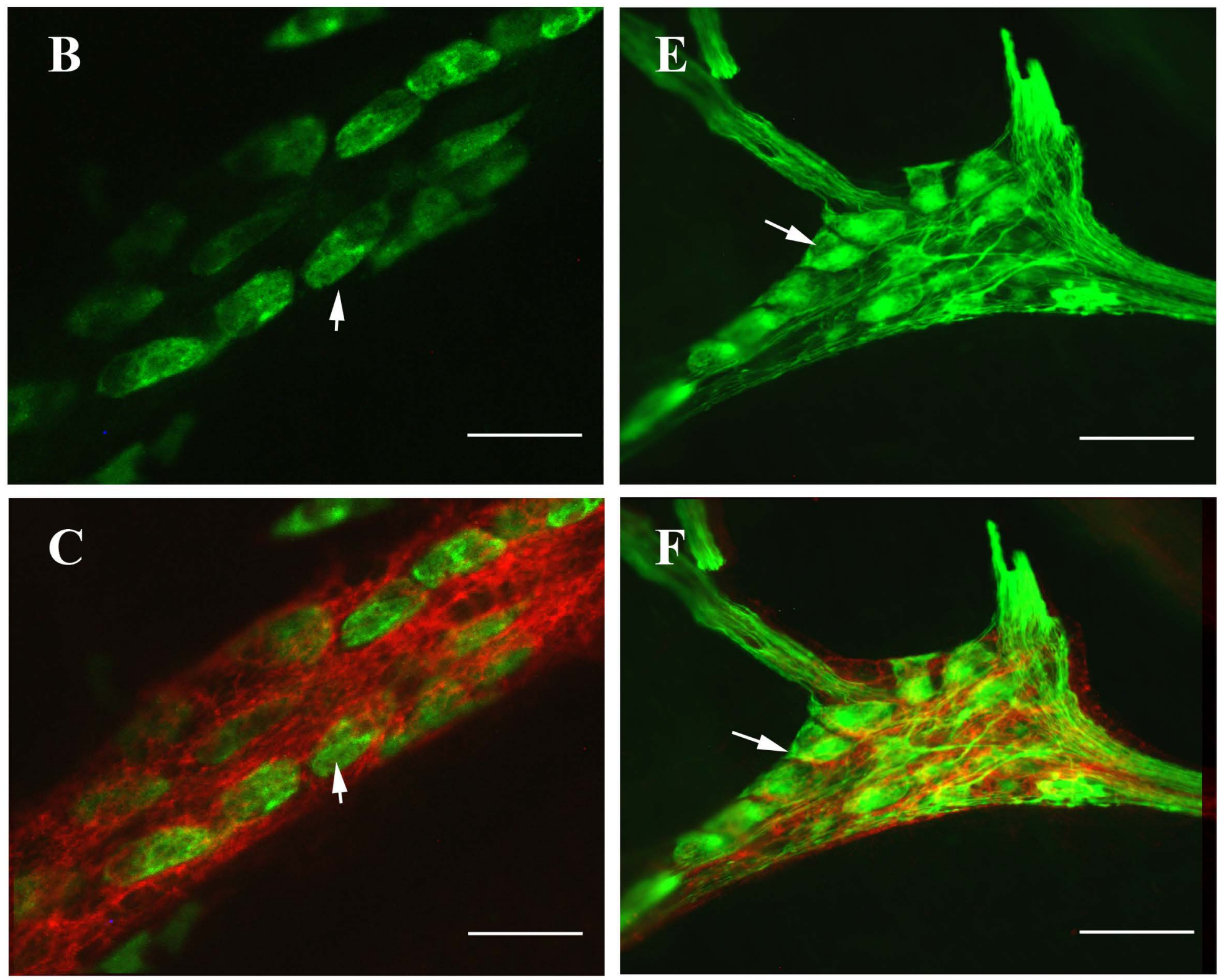
A

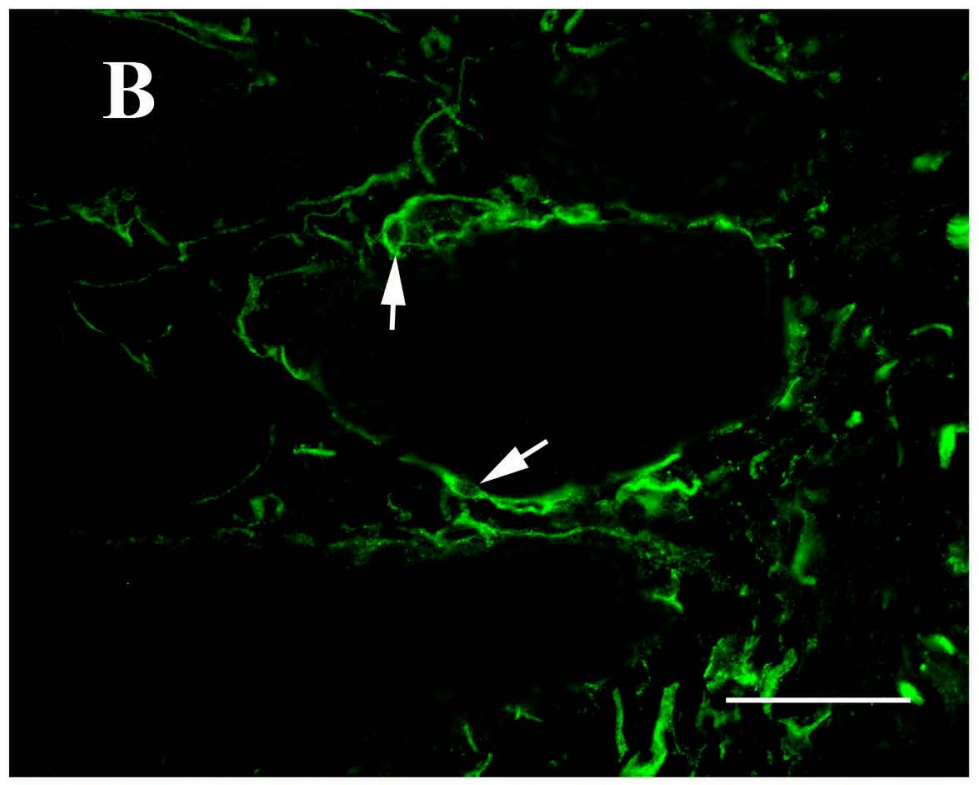

C

104

\section{$\checkmark$}

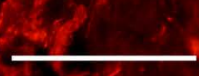

D

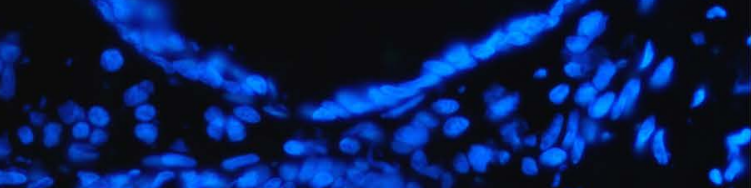
"Ifin

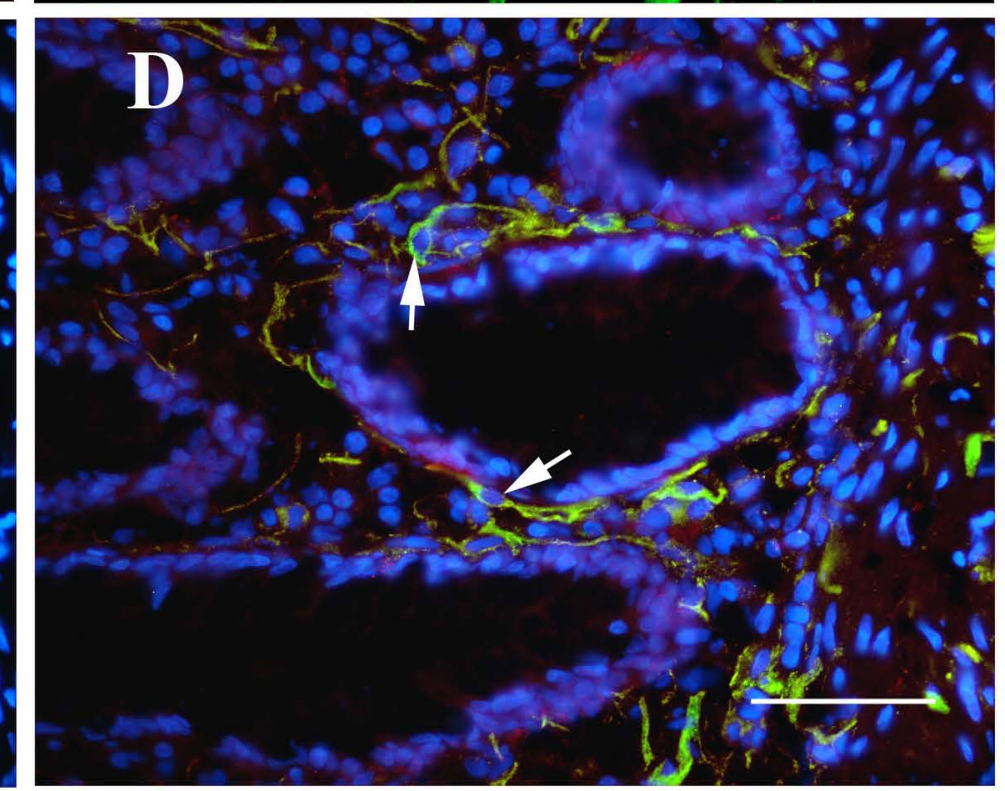

